\title{
Data Acquisition and Complex Systems Analysis in Critical Care: Developing the Intensive Care Unit of the Future
}

\author{
Frank J. Jacono, $\mathrm{MD}^{1,2}$, Michael A. De Georgia, $\mathrm{MD}^{3}$, \\ Christopher G. Wilson, PhD ${ }^{4,5}$, Thomas E. Dick ${ }^{1,5}$, PhD, \\ Kenneth A. Loparo, PhD ${ }^{6}$,* \\ ${ }^{1}$ Division of Pulmonary, Critical Care and Sleep Medicine, CWRU School of \\ Medicine and University Hospitals Case Medical Center \\ ${ }^{2}$ Division of Pulmonary, Critical Care and Sleep Medicine, Louis Stokes VA \\ Medical Center \\ ${ }^{3}$ Neurological Institute, Department of Neurology, CWRU School of Medicine \\ and University Hospitals Case Medical Center \\ ${ }^{4}$ Department of Pediatrics, CWRU School of Medicine \\ ${ }^{5}$ Department of Neurosciences, CWRU School of Medicine \\ ${ }^{6}$ Department of Electrical Engineering and Computer Science, \\ $C W R U$ School of Engineering
}

\begin{abstract}
Modern hospitals are equipped with sophisticated monitoring equipment that displays enormous volumes of raw data about the cardiopulmonary and neural functions of patients. The latest generation of bedside monitors attempts to present these data to the clinician in an integrated fashion to better represent the overall physiological condition of the patient. However, none of these systems are capable of extracting potentially important indices of pattern variability inherent within biological signals. This review has three main objectives. (1) To summarize the current state of data acquisition in the intensive care unit and identify limitations that must be overcome to achieve the goal of real-time processing of biological signals to capture subtleties identifying "early warning signals" hidden in physiologic patterns that may reflect current severity of the disease process and, more importantly, predict the likelihood of adverse progression and death or improvement and resolution. (2) To outline our approach to analyzing biological waveform data based on work in animal models of human disease. (3) To propose guidelines for the development, testing and implementation of integrated software and hardware solutions that will facilitate the novel application of complex systems approaches to biological waveform data with the goal of risk assessment.
\end{abstract}

Keywords: critical care, ICU, complex systems, monitoring, decision support

*Corresponding Author: Kenneth A. Loparo, PhD, Nord Professor of Engineering, Case Western Reserve University, 10900 Euclid Avenue, Cleveland, Ohio 44106-7071. Phone: 216.368.4115, Fax: 216.368.6888, E-mail: kenneth.loparo@case.edu 


\section{INTRODUCTION}

More than four million patients are admitted to intensive care units (ICUs) each year. Mortality rates average between 10-20\% and approximately 500,000 patients die in US ICUs each year [1-3]. Given the high stakes involved, timely and effective care is paramount, and this requires continuous patient surveillance using sophisticated monitoring equipment that gathers enormous volumes of raw physiological data. As a result, ICUs are complex, data-intense environments and the ability of clinical personnel to quickly detect subtle changes in patient physiology and to respond rapidly is crucial. Dozens of systemic parameters are monitored, including heart rate, respiration, arterial oxygen saturation, temperature, and end tidal $\mathrm{CO}_{2}$ concentration. Insertion of specialized catheters enables monitoring of arterial blood pressure, central venous pressure, pulmonary arterial pressure, right and left atrial pressure, as well as calculations of stroke volume, systemic and pulmonary vascular resistance, and cardiac output. Additional neuromonitoring that includes intracranial pressure, brain tissue oxygenation, cerebral blood flow, and continuous electroencephalography, is often superimposed on systemic monitoring [4]. New and improved instruments continue to be developed and incorporated in the ICU to monitor physiological parameters. Because biological organisms are innately complex dynamical systems, the effective integration of these data is essential to improving critical care in the ICU.

It is our hypothesis that the state of an individual (in health and disease) is reflected in the temporal patterning of physiological signals over time, and in the dynamic relationships between these signals $[5,6]$. Thus, we are proposing a paradigm shift in thinking where the analysis of dynamic (temporal) changes in physiologic signals and their relationship to organ system interconnections is as important (and possibly more important) than individual organ system function. The potential of studying nonlinear disease dynamics has been demonstrated in various clinical states such as sudden cardiac death after acute myocardial infarction, congestive heart failure, brain injury, sepsis, and the prediction of hypotension during dialysis [7-19]. However, techniques for the analysis of nonlinear systems from observed time series data have never been systematically applied to all the recorded physiological data simultaneously within the intensive care unit setting and never in real-time [20, 21]. Ultimately, we envision measures (linear stochastic/nonlinear deterministic) of pattern variability as "additional vital signs" that provide diagnostic and predictive information to clinicians. While initially applied in the intensive care unit, these approaches will be easily applied in other settings identifying patients in need of a higher level of care or more intensive therapy.

The latest generation of monitors attempts to integrate this diverse dataset to better represent the overall physiological condition of the patient, but typically this encompasses only means and data trends that are inadequate for managing complex biological systems in the ICU. As a result of proprietary data formats from instrument vendors, the ability to effectively use waveform data is hampered by difficulties in the real-time acquisition and time-synchronization of the raw physiological data. Therefore, despite the growth in the need for critical care and advances in the development of new measurement and sensing technology, the basic information technology infrastructure needed to support signal data acquisition, analysis, integration 
and management remains primitive and outdated [22, 23]. Significant challenges must be overcome to assure that next-generation monitors are capable of real-time processing of waveform data to capture subtleties identifying "early warning signals" hidden in physiologic patterns that reflect current severity of the disease process and, more importantly, to predict the likelihood of adverse progression and death or improvement and resolution.

In this review we will: 1) examine the current state of data acquisition in the ICU, 2) outline our approach to analyzing biological waveform data based on work in animal models of human disease, and 3) propose guidelines for the development, testing and implementation of integrated software and hardware solutions that will facilitate the novel application of complex systems approaches to biological data with the goal of risk assessment.

\section{CURRENT STATE OF ICU DATA ACQUISITION AND INTEGRATION}

The widespread use of patient monitoring was central to the evolution of critical care medicine as a specialty in the 1950s. By 1980, three major developments had occurred: (1) Pulmonary units were established to cope with the polio epidemics that had swept through the U.S. and Europe; (2) Coronary care units were established following the introduction of synchronous direct-current cardioversion; (3) Anesthesia critical care developed to co-manage complex surgical patients in surgical ICUs. These developments provided increased operating efficiency by grouping patients in a single location and spawned the development of specialized instrumentation such as the pulmonary artery catheter and continuous electrocardiographic monitoring of the heart.

Since the inception of critical care medicine, monitoring technology has advanced significantly. Although monitoring systems have improved, concomitant advances in data analysis have not. In most instances, caregivers laboriously log the parametric data from the monitor by hand onto paper charts or into electronic medical records with no significant processing or analysis of physiologic data beyond simple trending. With the growth of electronic medical records, many devices are capable of directly populating the medical record with monitored data. However, even when this is available, data are recorded at very slow (seconds or minutes) sampling rates as compared to the intrinsic dynamics of the signals being recorded. While this approach may provide general trend information, physiological signals have information content over much faster time scales. This information is obscured, lost and unrecoverable from data that is sampled below critical sampling rates.

Currently, the waveform physiologic data commonly collected in the ICU (electrocardiogram, respiration, blood pressure, etc...) are not integrated into a searchable and secure data archive. Although "full-disclosure" ICU monitoring systems are becoming more common, tools that translate physiological data streams into analyzable time series with high sampling rates are rare. The latest generation of commercially available monitors is capable of measuring multiple parameters at varying temporal resolutions (Table 1). Despite this, most bedside monitors numerically display $3-5 \mathrm{~s}$ time-averaged parametric data next to waveforms. Any processing that is performed is restricted to linear time series analysis using conventional statistics (e.g. 
Table 1. Vendor data export specifications

\begin{tabular}{|c|c|c|c|}
\hline Monitor & Measurement & Sample Rate & Communications \\
\hline \multirow[t]{21}{*}{ Philips IntelliVue [70] } & ABP waveform & 125 or $500 \mathrm{~Hz}$ & RS232 MIB \\
\hline & ECG waveform & 125 or $500 \mathrm{~Hz}$ & RS232 MIB \\
\hline & ICP waveform & 125 or $500 \mathrm{~Hz}$ & RS232 MIB \\
\hline & Pleth waveform & 125 or $500 \mathrm{~Hz}$ & RS232 MIB \\
\hline & Resp waveform & 125 or $500 \mathrm{~Hz}$ & RS232 MIB \\
\hline & ICP numeric & $0.98 \mathrm{~Hz}$ & RS232 MIB \\
\hline & CPP numeric & $0.98 \mathrm{~Hz}$ & RS232 MIB \\
\hline & EtCO2 numeric & $0.98 \mathrm{~Hz}$ & RS232 MIB \\
\hline & FiO2 numeric & $0.98 \mathrm{~Hz}$ & RS232 MIB \\
\hline & HR numeric & $0.98 \mathrm{~Hz}$ & RS232 MIB \\
\hline & MAP numeric & $0.98 \mathrm{~Hz}$ & RS232 MIB \\
\hline & RR numeric & $0.98 \mathrm{~Hz}$ & RS232 MIB \\
\hline & $\mathrm{SpO} 2$ numeric & $0.98 \mathrm{~Hz}$ & RS232 MIB \\
\hline & Temp numeric & $0.98 \mathrm{~Hz}$ & RS232 MIB \\
\hline & Tart numeric & $0.98 \mathrm{~Hz}$ & RS232 MIB \\
\hline & Tcore numeric & $0.98 \mathrm{~Hz}$ & RS232 MIB \\
\hline & Tesop numeric & $0.98 \mathrm{~Hz}$ & RS232 MIB \\
\hline & Tnaso numeric & $0.98 \mathrm{~Hz}$ & RS232 MIB \\
\hline & Trect numeric & $0.98 \mathrm{~Hz}$ & RS232 MIB \\
\hline & Tskin numeric & $0.98 \mathrm{~Hz}$ & RS232 MIB \\
\hline & Tven numeric & $0.98 \mathrm{~Hz}$ & RS232 MIB \\
\hline \multirow[t]{2}{*}{ Integra Licox [71] } & $\mathrm{PbtO} 2$ & $2 \mathrm{~Hz}$ & RS232 Serial - ASCII \\
\hline & ICT & $2 \mathrm{~Hz}$ & RS232 Serial - ASCII \\
\hline \multirow[t]{5}{*}{ Integra Camino [72] } & ICP wave & Approx $190 \mathrm{HZ}$ & RS232 Serial \\
\hline & ICP numeric & $1 \mathrm{~Hz}$ & RS232 Serial \\
\hline & MAP numeric & $1 \mathrm{~Hz}$ & RS232 Serial \\
\hline & $\mathrm{CPP}$ numeric & $1 \mathrm{~Hz}$ & RS232 Serial \\
\hline & ICT numeric & $1 \mathrm{~Hz}$ & RS232 Serial \\
\hline Somanetics INVOS [73] & RSO2 & $0.2 \mathrm{~Hz}$ & Serial Output \\
\hline Hemedex Bowman & Perfusion & $1 \mathrm{~Hz}$ & \\
\hline \multirow[t]{2}{*}{ Perfusion [74] } & Tperf & $1 \mathrm{~Hz}$ & RS232 Serial \\
\hline & $\Delta$ Tperf & $1 \mathrm{~Hz}$ & RS232 Serial \\
\hline \multirow[t]{3}{*}{ CSZ Blanketroll III [75] } & Tcore & $0.2 \mathrm{~Hz}$ & USB \\
\hline & Twater & $0.2 \mathrm{~Hz}$ & USB \\
\hline & TsetPt & $0.2 \mathrm{~Hz}$ & USB \\
\hline
\end{tabular}

mean, variance, correlation, coefficient of variation). Alarm limits are often based on simple threshold crossing of an instantaneous signal without regard for the dynamic interactions between physiological variables [24]. In a recent review of clinical decision support capabilities of commercially-available clinical information systems, analysis of waveform data was not available as "input data" for any of the systems [25]. 
This inability to integrate and time-synchronize physiologic signal data simultaneously into one dataset has been a major limiting factor in intensive care monitoring. The status quo has a negative impact on patient care and impedes clinical research studies. For example, attempts have been made to develop integrated knowledge-based systems for ventilator control and intelligent ICU monitoring [26, 27], but these are not widely used in part due to the inaccessibility of pertinent physiological waveform data streams. Furthermore, the fields of dynamical systems analysis and information theory have already developed techniques for measuring potentially important indices of pattern variability from biological signals, and there is a growing body of evidence that these measures may be clinically relevant [14, 16, 28-44].

The future of critical care will require "information management", which includes the real-time collection, integration, and interpretation of various types of physiological data from multiple sources, specifically, (1) the continuous integration and timesynchronization of multiple channels of physiological data, and (2) real-time data analysis and feature extraction using multivariate and nonlinear ("complex systems") time series analysis methods together with visualization tools designed to facilitate rapid diagnosis and treatment.

\section{APPROACH TO APPLYING COMPLEX SYSTEMS ANALYSIS TO BIOLOGICAL WAVEFORM DATA}

Biological systems are intrinsically nonlinear and thus have a degree of complex and chaotic behavior (seemingly random behavior from a deterministic system), which is often an intrinsic property of the normal, healthy state [45-47]. In fact, the complex pattern variability of physiological waveforms is often considered a hallmark of the normal physiological state and has two basic components: Stochastic variability in which the present state of the system does not completely determine succeeding states and fluctuations about the mean trend are random and unpredictable, and Deterministic variability in which the pattern includes temporal structure (often beyond that described by linear correlation) that exists across time. These dynamics can change in critical illness [6], and emerging evidence suggests that nonlinear changes in dynamics over time may have predictive value, facilitating earlier recognition of deterioration and more timely intervention resulting in better patient outcomes [13, 48-50]. Techniques for the analysis of nonlinear systems have emerged from the mathematical and engineering sciences but, for the most part, have not been systematically applied to physiological data in the ICU setting. Our fundamental hypothesis is that: Patient care in the ICU can be significantly improved through the application of complex system analysis methods to acquired and synchronized physiological signal data.

Traditional analyses fallaciously assume that all fluctuations and pattern variability are stochastic and fail to isolate and quantify deterministic sources of variability. We have developed a framework (Figure 1) for the analysis/interpretation of biological data using nonlinear dynamical systems techniques applied to the physiological time series measurements acquired at the appropriate sampling rates. Methods for complex systems analysis complement existing approaches, quantify temporal variability, and provide insight into the potential mechanisms of variability underlying physiological signals. 


\section{SOURCES OF VARIABILITY}

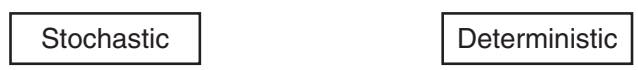

Conceptual Properties

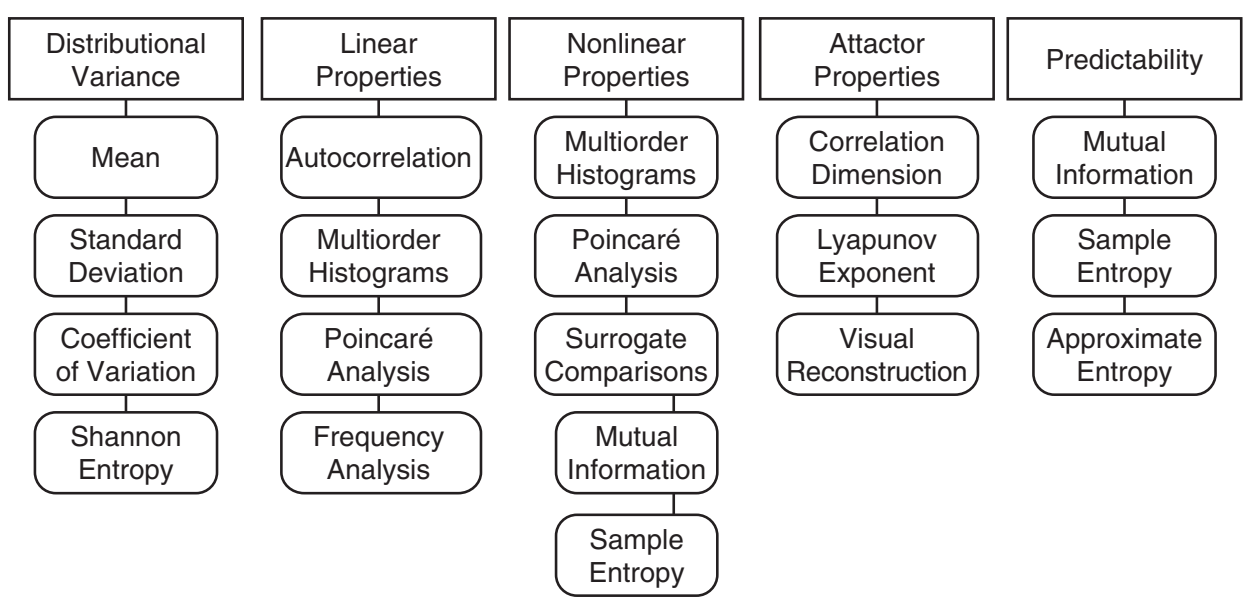

Figure 1. Conceptual properties describing biological variability and selected tools to quantify sources of variability.

While sources of both stochastic and deterministic variability contribute to system behavior, these determinants of variability cannot be measured directly, nor completely separated. However, through a consistent and disciplined approach incorporating multiple methods and suitable surrogate data analysis, a comprehensive understanding of signal variability and its relationship to health and disease can be achieved.

Our overall goal is to understand how different components of biological variability interact during health and disease using a balanced application of computational methods while recognizing the strengths, assumptions and limitations of each approach. For example, reliance on a single technique may lead to over-interpretation [51], whereas a comprehensive view enables the formation of mechanistic hypotheses that evaluate the sources of variability more fully. To help formalize this process, we have prepared a set of conceptual properties, each describing a unique facet of variability (Fig. 1):

Distributional Variance: Provides a measure of how variability is distributed independent of temporal dynamics. This property treats the signal as a stochastic process and uses standard measurements such as mean, standard deviation and Shannon Entropy.

Linear Properties: Characterizes linear relationships in the data in both the time and frequency domains. Measurement tools include autocorrelation, multiorder histograms, Poincaré plots (circle-return maps). 
Nonlinear Properties: Measures temporal relationships in the data that are not linear in nature. Surrogate comparisons of mutual information and sample entropy, as well as multiorder histograms and Poincaré analysis provide insight into nonlinear properties.

Attractor Properties: Reconstructs the attractor (if one exists) and characterizes invariant measures such as correlation dimension and Lyapunov exponents.

Predictability: Is related to both the linear and nonlinear properties in that it measures relationships, but more specifically characterizes the amount of information that is contained in the measurements and characterizes the time series from an information theory perspective.

It is important to realize that although variability by itself is a useful concept, the mechanism that is responsible for the variability may also be important. Stochastic and deterministic variability are two types of variability that may result from independent physiological sources. The facets are neither independent nor comprehensive; they are meant as suggestive of the types of variability that can be quantified in time series data and that we have observed to be of interest in many of our studies. Linear deterministic systems are "predictable" and cannot produce the type of variability that can result from either a stochastic or nonlinear system. Therefore the major issue is to distinguish between linear stochastic and nonlinear sources of variability. The list of analytical tools associated with each conceptional property is far from comprehensive, and is meant only to illustrate the breadth of techniques that can provide insight into varous facets of signal varibility. We now briefly summarize some important analytical techniques for signal analysis that are part of our stepwise approach:

(1) Selection of stationary epochs to minimize the influence of other physiologic factors that could influence dynamics.

(2) Conventional Analysis including mean, standard deviation (SD), coefficient of variation $(\mathrm{CV})$ and frequency domain analysis of periodic signals like cardiac activation or breathing pattern.

(3) Assessment of pattern morphology: For periodic signals, the morphology and variability of each cycle can be different. For example, breathing pattern morphology will not be completely captured by an analysis of phase durations (e.g., $\mathrm{T}_{\mathrm{I}}=$ time of inspiration, $\mathrm{T}_{\mathrm{E}}=$ time of expiration and $\mathrm{T}_{\mathrm{TOT}}=$ total duration of respiration). While part of the observed variation is due to noise, deterministic changes in the morphology of the respiratory pattern may reflect alterations in the processing of afferent inputs by the cardiorespiratory network.

(4) Multiorder histograms capture all sources of variability, show periodic distribution of data and give an intuitive display of data dependence.

(5) Autocorrelation functions constructed across a range of time delays $(\tau)$ assess linear dependence in the data set.

(6) Mutual information (MI) quantifies statistical dependence in the data set by assessing how the uncertainty of a time-shifted coordinate $\mathrm{x}(\mathrm{t}+\tau)$ is influenced by the knowledge of a coordinate in the original data set $x(t)$.

(7) Poincaré analysis examines the relationship between cycles in periodic signals and determines the relationship between switching behaviors. We generalize the Poincaré construction as $\mathrm{n} v s . \mathrm{n}+\mathrm{d}$ and compare how the variability measurements change across delays (d) and in relationship to changes in underlying physiology. 
(8) Sample entropy (SampEn) is a measure of self-similarity in a time series [52, 52] with lower values indicating more self-similarity, lower complexity and less variability. SampEn is initially computed with standard parameters [54]: pattern length $\mathrm{m}=3$ points, tolerance $\mathrm{r}=0.2 *$ Standard Deviation, and time delay $\tau=1$. To reveal additional temporal patterns in the data $\mathrm{m}$ is varied, and computations across different values of $\tau$ for each epoch are used to explore nonlinear contributions to pattern complexity [55].

(9) Surrogate data testing investigates the mechanisms generating variability [56]. Surrogate data sets are computed using the iterated amplitude adjusted Fourier transform (iAAFT) which moves data into the frequency domain for adjustment and then back into the time domain while ensuring that both the frequency distribution (power spectrum/autocorrelation function) and the amplitude distribution are maintained [57]. Differences between the MI or SampEn of the surrogates and those of the original data are used as an index of the amount of nonlinear complexity present in the data for a given value of $\tau$.

(10) Correlation dimension (D2) quantifies complexity and is an approximate measure of the number of active degrees of freedom of the system [58]. D2 can be used to differentiate between stochastic and deterministic sources of variability, as D2 can only be calculated when levels of stochastic variability are very close to zero. An inability to obtain a stable measure of D2 is interpreted to mean that significant stochastic variability is present.

(11) Integrate Results into a Comprehensive Picture: Once a full analysis has been completed, the different results are studied in a complementary fashion. Biological systems are highly complex and capable of displaying a wide variety of behaviors. In this regard, features that capture both the linear stochastic and nonlinear deterministic aspects of the signal are important. Thus comprehensive analysis integrates multiple techniques in order to understand how changes in stochastic and deterministic variability are applicable to changes in patient status.

\section{BREATHING PATTERN VARIABILITY AS A POTENTIAL PREDICTOR OF MORTALITY}

The raw data (Figures 2A, 2B, and 2C) are plethysmographic recordings and depict respiratory patterns from awake rats breathing room air on 1, 5, and 9 days after the induction of chemically-induced acute lung injury (ALI). These three selected animals include: an ALI animal that died on the $6^{\text {th }}$ day after bleomycin instillation (Figure 2A); an ALI animal that recovered with eventual return to normal weight gain and no evidence of lung fibrosis as measured by lung collagen content at two weeks after initial injury (Figure 2B); and a 'control' animal that received sham surgery with intratracheal instillation of saline rather than bleomycin (Figure 2C). Breathing patterns were assessed in all animals at each time point. Respiratory rate increased in both animals with ALI, but the ALI survivor maintained more temporal pattern variability in its breathing pattern when compared to the animal with lethal ALI. The decreased variability in the lethal ALI animal is evident in the raw record, particularly at day 5 (Compare Figures 2A and 2B). Even though frequency remained elevated on the $9^{\text {th }}$ day in the ALI survivor, variability returned to a level similar to that observed in the sham surgery control (Compare bottom traces of Figures $2 \mathrm{~B}$ and $2 \mathrm{C}$ ). Properties of the 


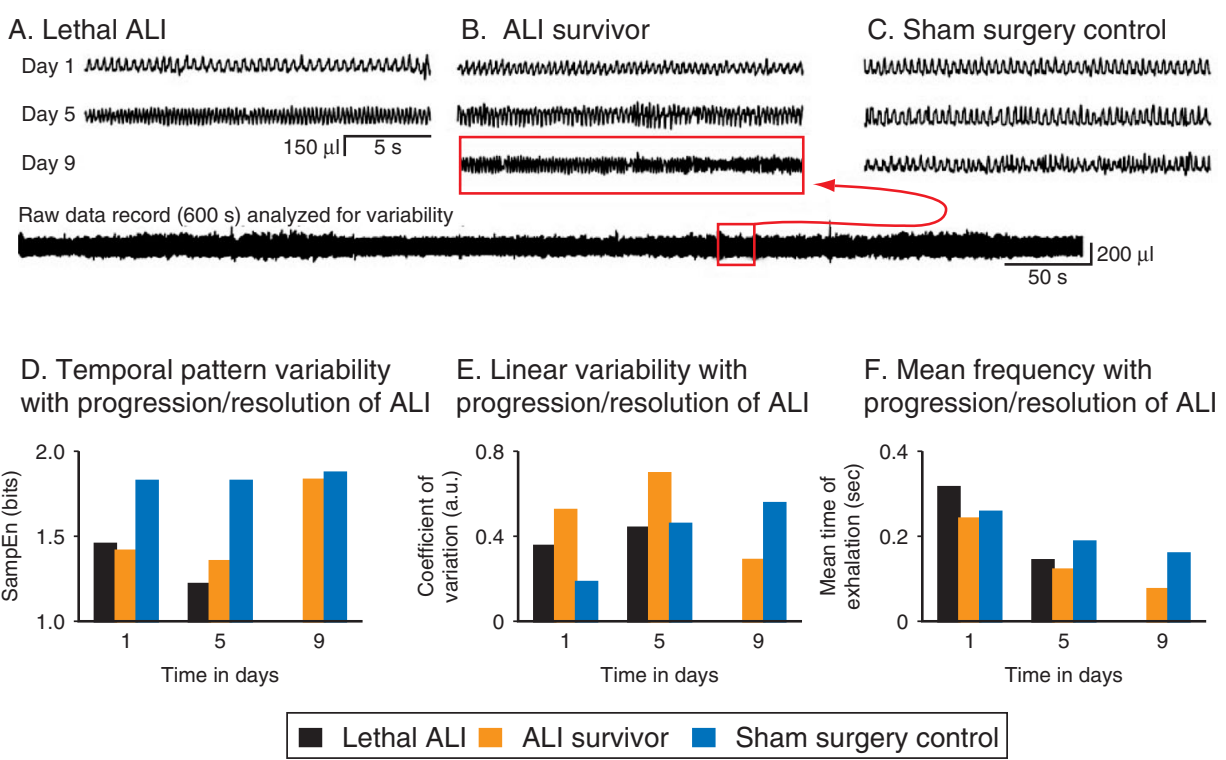

Figure 2. Breathing pattern variability as a predictor of mortality.

breathing pattern are quantified in Figures 2D, 2E, and 2F. Despite the increase in respiratory rate, the ALI survivor maintained a moderate level of variability, a harbinger of its eventual recovery. Entropy measurements quantified an increase in predictability as a potential marker of eventual mortality. Specifically, temporal pattern variability of the breathing pattern was quantified by the analysis of successive differences in the duration of expiration across the data set (Figure 2D). The standard deviation time series of the successive differences is analyzed using SampEn across time lags determined by mutual information for all animals across all time points and reveals the following: a) Sham surgery control (blue bars) had the least predictable (most complex; highest entropy) breathing pattern at all time points; b) The animal with lethal ALI (black bars) had the greatest predictability on the day prior to death; c) The ALI survivor (orange bars) had increased predictability of breathing pattern during acute lung injury but demonstrated evidence of recovery by day 9 with entropy measurements similar to the saline control. In contrast, coefficient of variation of time of exhalation was unable to discriminate between these three conditions (Figure 2E). For example, the lethal ALI animal had similar coefficient of variation to the sham surgery control on the day prior to death. Trend data for respiratory frequency (Figure 2F) as quantified by mean time of exhalation identified changes at day 5; however, this measure was not able to identify at day 1 animals that would go on to develop ALI. While anecdotal, these results suggest that measures of breathing pattern variability should be investigated further as potential predictive tools for risk assessment. 


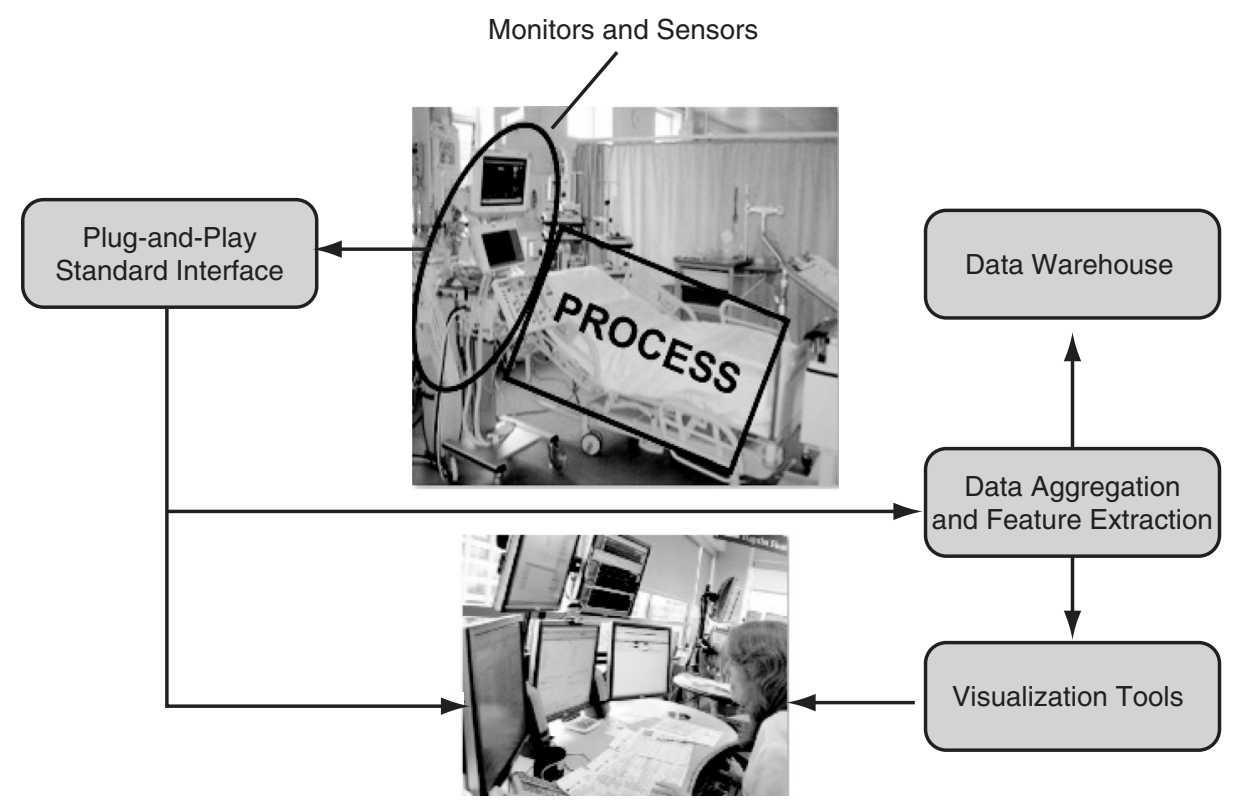

Figure 3. Development of distributed data acquisition and control process for data acquisition in the ICU.

Our approach to developing human patient data acquisition at the bedside in the ICU is based on the development of distributed data acquisition and control systems in the process in manufacturing industries, as illustrated in Figure 3. In our model, the patient is the process and all the ICU instruments are connected to a data highway through a plug-and-play sensor interface module that provides information to a monitor for standard trending. In addition, unfiltered waveforms are passed to a data aggregation and feature extraction module where information from physiological signals can be analyzed, integrated, and presented to the intensive care staff using novel visualization tools. Data archived in a secure data warehouse can then be merged with electronic medical record data to provide a more complete dataset that is available to the ICU team and other caregivers. As part of our ongoing study, we have collected data using commercial ICU monitors and Rugloop II software (Demed, Belgium). Preliminary feature extraction was used to quantify temporal pattern variability in respiration and heart rate as shown in Figures 4, 5, and 6. Figure 4 (ECG, Breathing and EEG verses time in seconds) illustrates the simultaneous acquisition of real-time data including automatic identification of R-waves and inspiration and expiration times, and in Figures 5 and 6, Poincaré analyses of heart rate (R-R) and respiratory (inspiration) variability reveal a change in the temporal structure of variability as a function of delay $d$. 

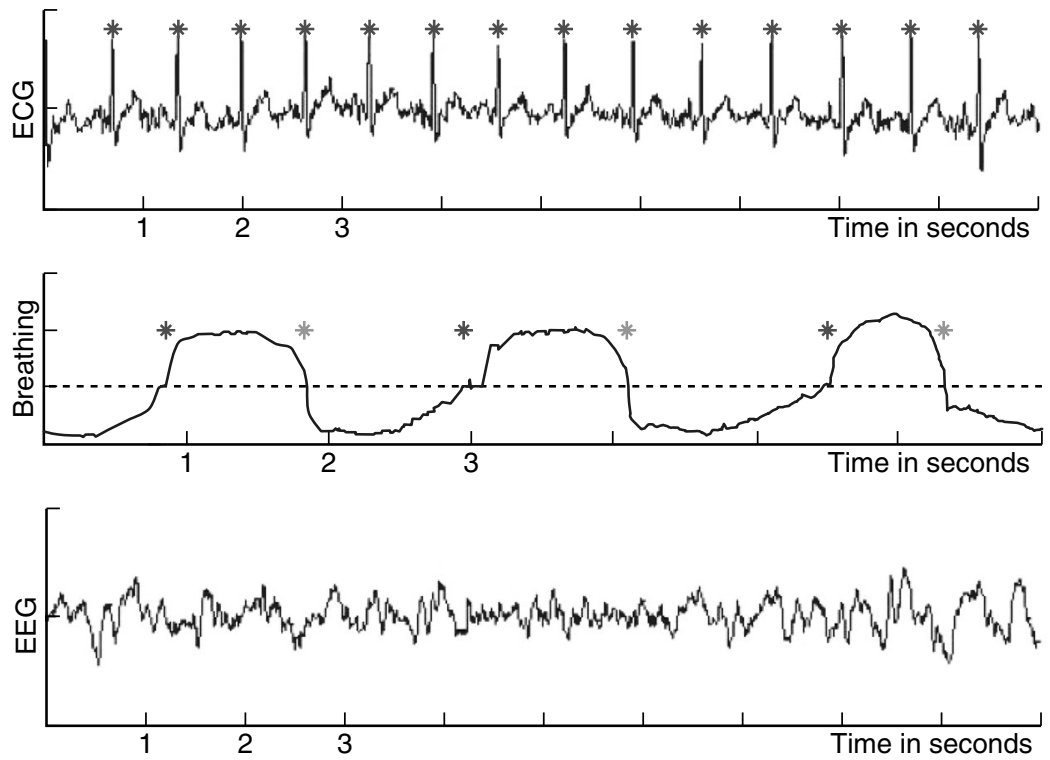

Figure 4. Sample Data with automatically detected R- waves, Inspiration and Expiration times.
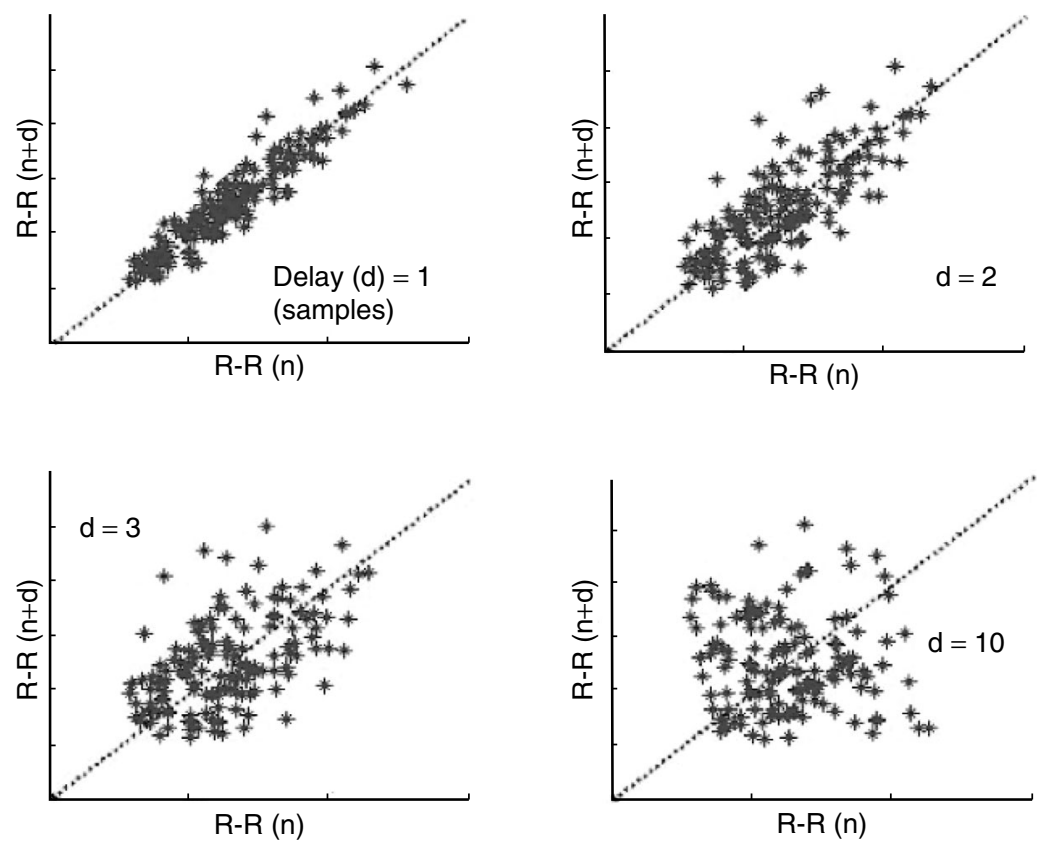

Figure 5. Poincaré plot of R-R intervals with different delays (d). 

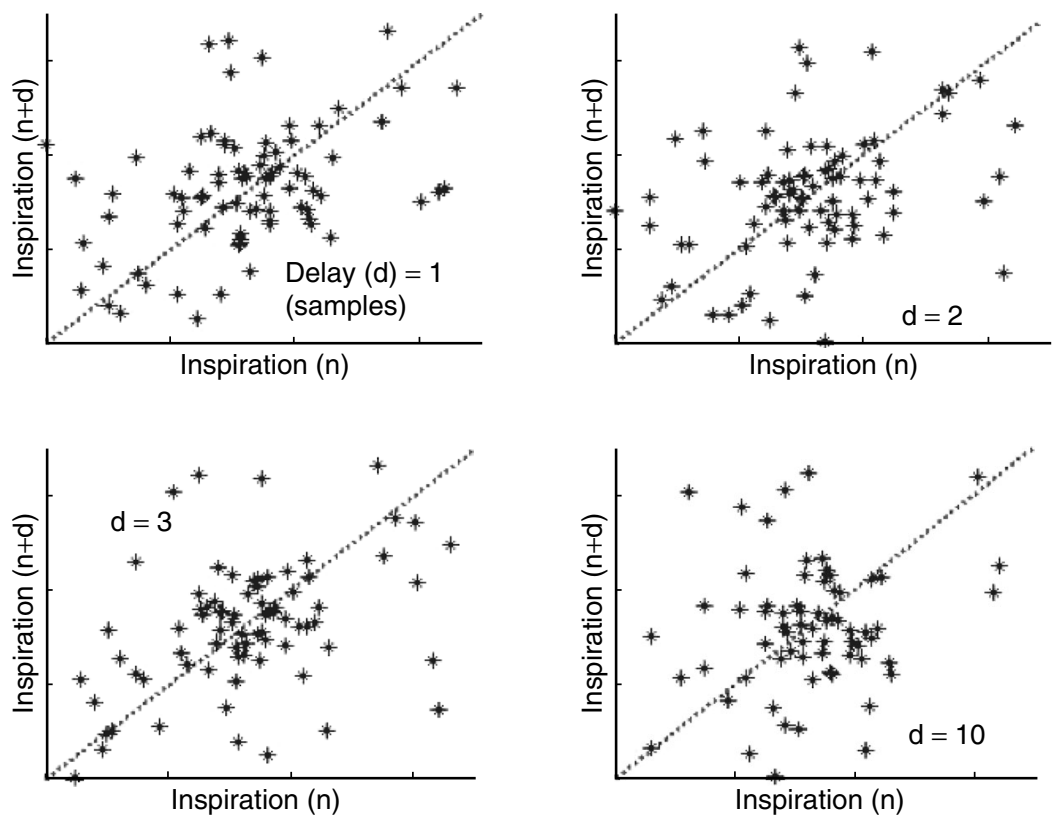

Figure 6. Poincaré plot of time difference between two consecutive inspiration events with different delays (d).

\section{DISCUSSION}

Beyond simple trending, there is a dearth of processing or analysis of physiological signal data in current ICU practice. Because most physiological signals include both linear stochastic- and nonlinear deterministic-features, the development and deployment of modeling methods that can effectively and efficiently use both types of information in characterizing phenotype differences based on these measured variables is required. The medical field has traditionally used linear statistical techniques to approximate trends in biological data and, although these techniques are useful, they are limited in their applicability to complex biological systems that are intrinsically nonlinear and exhibit complex behavior.

The analysis of variability and other methods of feature extraction of biological waveform data have the potential to provide unique and complementary information for clinical decision-making. Pattern variability in biological signals is an intrinsic property of the normal, healthy state [60]. There is a "sweet spot" for physiologic time series variability as a biomarker for disease. In this context, too little variability and too much variability are indicators of "poor health." Further, changes in variability occur pathologically in critical illness, and recovery from critical illness is characterized by recovery of the inherent variability of the healthy state [30, 61-67]. This information regarding variability is "hidden" in commonly monitored physiologic signals and may provide important information about interconnections between organ systems, interdependencies and feedback relationships, and the overall physiology of the patient. 
Here we have proposed a comprehensive algorithmic framework for the analysis and interpretation of ICU data using nonlinear dynamic systems techniques applied to time series measurements. Complex systems analysis methods are complementary to existing approaches, can quantify temporal variability, and provide insight into potential variability mechanisms in physiological signals. On a mechanistic level, stochastic and deterministic sources of variability contribute to system behavior, but directly measuring and separating these sources is challenging. Through a consistent and disciplined approach incorporating complementary methods, improvements in understanding signal variability and its relationship to health and disease can be achieved. Because these approaches can capture the subtleties of dynamic physiological control systems in both health and disease they can provide useful insight into disease onset, tracking disease progression and the deployment of novel and more effective therapeutic interventions in the ICU.

The main obstacle to providing this enhanced information set is the availability of appropriately acquired (sampling rate, resolution, and time-synchronization) data. In the standard ICU setup it has been nearly impossible to acquire and integrate a diverse set of physiological data into one dataset for subsequent analysis. Even acquiring physiological waveform data and performing basic linear time series analysis using conventional statistics is, currently, frustratingly difficult. A fresh approach to developing patientcentered cognitive support for critical care is needed. Using mathematical models of physiologic subsystems to map clinical observations to testable hypotheses about physiologic conditions has the potential to improve insight into current patient status and, eventually, to predict responses to therapeutic interventions and to forecast more precisely individual patient trajectories [59]. However, this will require the development of tools for data acquisition, integration, time-synchronization, and analysis (using a full array of complex systems analysis methods).

The medical device industry has not incorporated many of the advances in computer science, biomedical engineering, signal processing, and mathematics into new products for the ICU. Thus, ICUs have yet to realize the vision of full integration of instrumentation, patient records, decision-support and reference tools, as well as their general computing and communication resources [9]. Several challenges need to be overcome before measures of variability can be incorporated into healthcare decision-making:

- Proprietary restrictions on data acquisition including but not limited to nonuniform irregular sampling of time-averaged physiologic data for diagnosis, prognosis and clinical decision-making.

- Insufficient computational power and a lack of specialized software.

- Incompatibility between monitoring equipment and systems for data collection and analysis, as well as limits to patient data storage.

- $\quad$ True plug-and play interoperability of all system components, both software and hardware. This is a key to future development of the ICU and a paradigm shift in patient care [9].

- $\quad$ Need to fully demonstrate and quantify the value of these advanced measures and biomarkers extracted from physiological time series data in facilitating clinical decision-making and improving clinical care. 
- $\quad$ Removing financial constraints that inhibit companies from adding advanced signal analysis to existing software. The availability of an improved information set has the potential to add value compared to conventional management of ICU information, but the cost/benefit tradeoff question remains unanswered. Unfortunately, the data needed to provide such a comparative analysis is not available at this time.

All of these problems should be surmountable. However, a complete overhaul of the basic information technology infrastructure in the ICU is required. We need standardized systems for data acquisition, integration, and real-time data analysis, and clear, user-friendly visualization tools incorporating decision support software to assist clinicians. A coordinated effort involving multi-institutional collaborations of clinicians, engineers, computer scientists, and experts in informatics and complex systems analysis, as well as industry is required to decisively move this emerging field of "critical care informatics" forward. As summarized in Figure 7, a multi-pronged approach is necessary:

1) Develop an open source information architecture, consistent with the Integrated Clinical Environment (ICE) architecture that supports the acquisition, timesynchronization and archiving of physiological wave form data from the ICU.

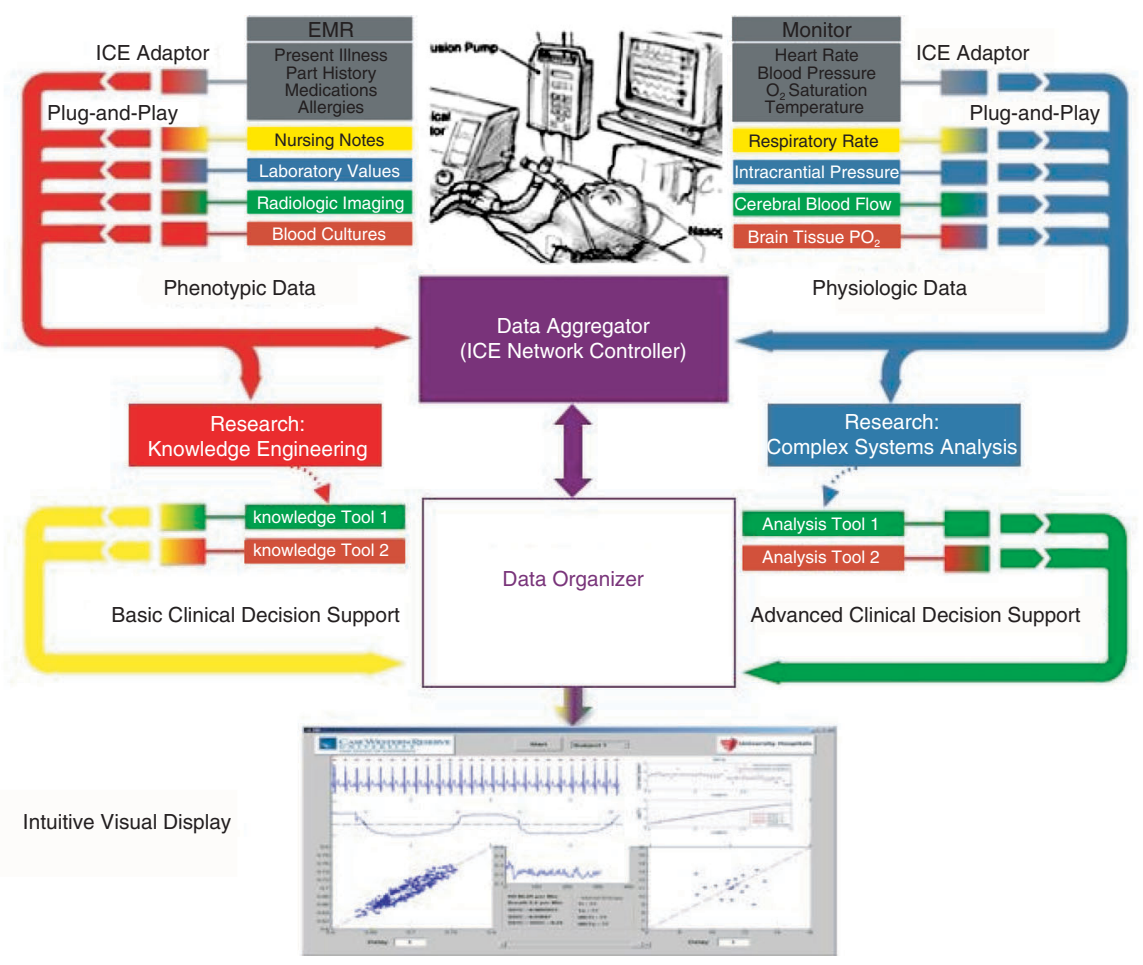

Figure 7. Phased approach to developing the ICU of the Future. 
2) Simultaneously develop computational models and algorithms using animal laboratory data to investigate which measures have the greatest promise for improving real-time clinical decision-making in the ICU.

3) Design and implement human research and clinical studies that can provide the necessary data to reliably address the utility (cost verses benefits) of the proposed approach.

The potential payoffs are great; the routine availability of this information would fundamentally change the way medicine is practiced, improving risk stratification, evaluations of efficacy, quality of care assurance, and ultimately patient outcome. In addition, this technology would be applicable to Tele-ICU networks capable of exporting expertise to hospitals and intensive care units where current personnel resource limits make the highest quality of care challenging.

\section{CONCLUSIONS}

The amount of data available to the clinician at the bedside has grown tremendously because of advances in medical monitoring and imaging technology. This situation is particularly evident in the critical care setting in which patients may be continuously monitored for upwards of 30 different variables related to multiple, changing and interacting complex physiological subsystems. Treatments are often titrated on a minute-to-minute basis. Taken together, this has created an information overload at the bedside that is overtaxing human capabilities to cope with large amounts of data $[68,69]$. The solution is to improve organization, analysis and presentation of monitored variables in the ICU. We have reached the limit of currently available methods of data acquisition to leverage complex systems analysis towards this goal. The limitations of the current approach are underscored by the fact that an improved capacity to acquire quantitative measurements highly relevant for therapeutic decision-making has failed to improve outcome. This failure is related primarily to our inability to acquire and integrate the sheer volume of available data, and to transform these data into information that can ease the cognitive burden of clinical personnel in interpreting the complicated and often nonlinear interactions of the various physiologic subsystems. While electronic charting systems are an improvement over paper records, they generally just recapitulate the record rather than provide additional integration or analysis.

In summary, the "ICU of the Future" will be based on an information architecture that supports plug-and-play interoperability with ICU instruments for data acquisition, specialized application modules for data processing and advanced signal analysis, and interactive visualization and decision support modules that facilitate the display and interpretation of multimodal physiologic data for ICU decision-making. To achieve this goal, we advocate a phased approach where the first step is to make all available trend data electronically available for clinical decision support. The second phase is to move from trend data to high resolution time-synchronized waveform data that can be integrated, archived and analyzed using both conventional and advanced signal processing methods. The third phase is to develop visualization and decision support tools that will transform data to useful information made available to clinical personnel in real-time to support decision-making and improve situational awareness. 


\section{ACKNOWLEDGEMENTS}

This work was supported by the NIH (HL 087377) and the Veterans Administration Research Service. The authors would like to thank Farhad Kaffashi, Ryan Foglyano and Mikkel Fishman for their participation in the development of the analytical approach, programming and data analysis. We also thank Dick Moberg of Moberg Research, Inc. for providing the technical specifications listed in Table 1.

\section{REFERENCES}

[1] Dasta JF, McLaughlin TP, Mody SH, Piech CT. Daily cost of an intensive care unit day: the contribution of mechanical ventilation. Crit Care Med 2005;33:1266-71.

[2] Zimmerman JE, Wagner DP, Draper EA, Wright L, Alzola C, Knaus WA. Evaluation of acute physiology and chronic health evaluation III predictions of hospital mortality in an independent database. Crit Care Med 1998;26:1317-26.

[3] Lwin AK, Shepard DS. Estimating Lives and Dollars Saved from Universal Adoption of the Leapfrog Safety and Quality Standards: 2008 Update. Washington, D.C.: The Leapfrog group; 2008.

[4] De Georgia MA, Deogaonkar A. Multimodal Monitoring in the Neurological Intensive Care Unit. The Neurologist 2005;11:45-54.

[5] Ahmad S, Tejuja A, Newman KD, Zarychanski R, Seely AJ. Clinical review: A review and analysis of heart rate variability and the diagnosis and prognosis of infection. Crit Care 2009;13:232.

[6] Buchman TG. Physiologic stability and physiologic state. J Trauma 1996;41:599-605.

[7] Pados BF, Thoyre SM, Carlson BW, Nix B. Heart Rate Variability as an Indicator of Physiologic Stability During Feeding in the Preterm Infant. Advances in Neonatal Care 2009;9:85-6 10.1097/01.ANC.0000350113.45742.17.

[8] Askanazi J, Silverberg PA, Hyman AI, Rosenbaum SH, Foster R, Kinney JM. Patterns of ventilation in postoperative and acutely ill patients. Crit Care Med 1979;7:41-6.

[9] Frassica JJ. CIS: where are we going and what should we demand from industry? J Crit Care 2004;19:226-33.

[10] Belair J, Glass L, An Der Heiden U, Milton J. Dynamical disease: Identification, temporal aspects and treatment strategies of human illness. Chaos 1995;5:1-7.

[11] Buchman TG, Cobb JP, Lapedes AS, Kepler TB. Complex systems analysis: a tool for shock research. Shock 2001;16:248-51.

[12] Goldstein B, Buchman TG. Heart rate variability in intensive care. J Intensive Care Med 1998;13:252-65.

[13] Goldstein B, McNames J, McDonald BA, et al. Physiologic data acquisition system and database for the study of disease dynamics in the intensive care unit. Crit Care Med 2003;31:433-41.

[14] Kleiger RE, Miller JP, Bigger JT, Jr., Moss AJ. Decreased heart rate variability and its association with increased mortality after acute myocardial infarction. Am J Cardiol 1987;59:256-62.

[15] Martin GJ, Magid NM, Myers G, et al. Heart rate variability and sudden death secondary to coronary artery disease during ambulatory electrocardiographic monitoring. Am J Cardiol 1987;60:86-9.

[16] Odemuyiwa O, Malik M, Farrell T, Bashir Y, Poloniecki J, Camm J. Comparison of the predictive characteristics of heart rate variability index and left ventricular ejection fraction for all-cause mortality, arrhythmic events and sudden death after acute myocardial infarction. Am J Cardiol 1991;68:434-9.

[17] Pelosi G, Emdin M, Carpeggiani C, et al. Impaired sympathetic response before intradialytic hypotension: a study based on spectral analysis of heart rate and pressure variability. Clin Sci (Lond) 1999;96:23-31.

[18] Singer DH, Martin GJ, Magid N, et al. Low heart rate variability and sudden cardiac death. J Electrocardiol 1988;21 Suppl:S46-55. 
[19] Tsui FC, Li CC, Sun M, Sclabassi RJ. Acquiring, modeling, and predicting intracranial pressure in the intensive care unit. Biomed Eng Applicat Basis Commun 1996;8:566-78.

[20] Glass L, Kaplan D. Time series analysis of complex dynamics in physiology and medicine. Med Prog Technol 1993;19:115-28.

[21] Goldberger AL. Applications of chaos to physiology and medicine. In: Kim JH, Stringer J, eds. Applied Chaos. New York: Wiley-Interscience; 1992:321-31.

[22] Craft RL. Trends in technology and the future intensive care unit. Crit Care Med 2001;29:N151-8.

[23] Hanson CW. Ticker tape medicine. Crit Care Med 2004;32:2551-2.

[24] Imhoff M, Kuhls S, Gather U, Fried R. Smart alarms from medical devices in the OR and ICU. Best Pract Res Clin Anaesthesiol 2009;23:39-50.

[25] Wright A, Sittig DF, Ash JS, Sharma S, Pang JE, Middleton B. Clinical decision support capabilities of commercially-available clinical information systems. J Am Med Inform Assoc 2009;16:637-44.

[26] Hayes-Roth B, Uckun S, Larsson JE, et al. Guardian: an experimental system for intelligent ICU monitoring. Proc Annu Symp Comput Appl Med Care 1994:1004.

[27] Dojat M, Brochard L, Lemaire F, Harf A. A knowledge-based system for assisted ventilation of patients in intensive care units. Int J Clin Monit Comput 1992;9:239-50.

[28] Ahmad S, Ramsay T, Huebsch L, et al. Continuous multi-parameter heart rate variability analysis heralds onset of sepsis in adults. PLoS One 2009;4:e6642.

[29] Casaseca-de-la-Higuera P, Simmross-Wattenberg F, Martin-Fernandez M, Alberola-Lopez C. A multichannel model-based methodology for extubation readiness decision of patients on weaning trials. IEEE Trans Biomed Eng 2009;56:1849-63.

[30] Burykin A, Buchman TG. Cardiorespiratory Dynamics During Transitions Between Mechanical and Spontaneous Ventilation in Intensive Care. Complexity 2008;13:40-59.

[31] Casolo G, Balli E, Taddei T, Amuhasi J, Gori C. Decreased spontaneous heart rate variability in congestive heart failure. Am J Cardiol 1989;64:1162-7.

[32] Goldberger AL. Heartbeats, hormones, and health: is variability the spice of life? Am J Respir Crit Care Med 2001;163:1289-90.

[33] Huikuri HV, Makikallio TH, Peng CK, Goldberger AL, Hintze U, Moller M. Fractal correlation properties of R-R interval dynamics and mortality in patients with depressed left ventricular function after an acute myocardial infarction. Circulation 2000;101:47-53.

[34] Lishner M, Akselrod S, Avi VM, Oz O, Divon M, Ravid M. Spectral analysis of heart rate fluctuations. A non-invasive, sensitive method for the early diagnosis of autonomic neuropathy in diabetes mellitus. J Auton Nerv Syst 1987;19:119-25.

[35] Mietus JE, Peng C, Ivanov PC, Goldberger AL. Detection of obstructive sleep spnea from cardiac interbeat interval time series. Computers in Cardiology 2000;27:753-6.

[36] Stein PK, Domitrovich PP, Huikuri HV, Kleiger RE. Traditional and nonlinear heart rate variability are each independently associated with mortality after myocardial infarction. J Cardiovasc Electrophysiol 2005;16:13-20.

[37] Tapanainen JM, Thomsen PE, Kober L, et al. Fractal analysis of heart rate variability and mortality after an acute myocardial infarction. Am J Cardiol 2002;90:347-52.

[38] Imhoff M, Bauer M. Time series analysis in critical care monitoring. New Horiz 1996;4:519-31.

[39] Massagram W, Boric-Lubecke O, Macchiarulo L, Chen M. Heart Rate Variability Monitoring and Assessment System on Chip. Conf Proc IEEE Eng Med Biol Soc 2005;7:7369-72.

[40] Papaioannou VE, Maglaveras N, Houvarda I, Antoniadou E, Vretzakis G. Investigation of altered heart rate variability, nonlinear properties of heart rate signals, and organ dysfunction longitudinally over time in intensive care unit patients. J Crit Care 2006;21:95-103; discussion -4.

[41] Seely AJ, Christou NV. Multiple organ dysfunction syndrome: exploring the paradigm of complex nonlinear systems. Crit Care Med 2000;28:2193-200. 
[42] Seely AJ, Macklem PT. Complex systems and the technology of variability analysis. Crit Care 2004;8:R367-84.

[43] Tibby SM, Frndova H, Durward A, Cox PN. Novel method to quantify loss of heart rate variability in pediatric multiple organ failure. Crit Care Med 2003;31:2059-67.

[44] Wang X, Chen M, Macchiarulo L, Boric-Lubecke O. Fully-integrated heart rate variability monitoring system with an efficient memory. Conf Proc IEEE Eng Med Biol Soc 2006;1:5064-7.

[45] Capra F. The Web of Life: A New Scientific Understanding of Living Systems. New York: Anchor Books, Doubleday; 1996.

[46] Goldberger AL, West BJ. Chaos in Physiology: Health or Disease? In: Hans Degn AVH, Lars F. Olsen, ed. Chaos in biological systems: Springer; 1987.

[47] West BJ. Fractal physiology and chaos in medicine. New Jersey: World Scientific, 1990; 1990.

[48] Buchman TG. Nonlinear dynamics, complex systems, and the pathobiology of critical illness. Curr Opin Crit Care 2004;10:378-82.

[49] Goldstein B, Fiser DH, Kelly MM, Mickelsen D, Ruttimann U, Pollack MM. Decomplexification in critical illness and injury: relationship between heart rate variability, severity of illness, and outcome. Crit Care Med 1998;26:352-7.

[50] Sorani MD, Hemphill JC, 3rd, Morabito D, Rosenthal G, Manley GT. New approaches to physiological informatics in neurocritical care. Neurocrit Care 2007;7:45-52.

[51] Webber CL, Jr. The meaning and measurement of physiologic variability? Crit Care Med 2005;33:677-8.

[52] Pincus SM, Goldberger AL. Physiological time-series analysis: what does regularity quantify? Am J Physiol 1994;266:H1643-56.

[53] Richman JS, Moorman JR. Physiological time-series analysis using approximate entropy and sample entropy. Am J Physiol Heart Circ Physiol 2000;278:H2039-49.

[54] Pincus S. Approximate entropy (ApEn) as a complexity measure. Chaos 1995;5:110-7.

[55] Kaffshi F, Foglyano R, Wilson CG, Loparo KA. The effect of time delay on Approximate \& Sample Entropy calculations. Physica D: Nonlinear Phenomena 2008; 237 3069-74.

[56] Schreiber T, Schmitz A, . Surrogate time series. Physica D 2000;142:346-82.

[57] Kantz H, Schreiber T. Nonlinear Time Series Analysis. 2nd. ed: Cambridge University Press; 2004.

[58] Grassberger P, Procaccia I. Measuring the strangeness of strange attractors. Physica D 1983;9:189-208.

[59] Lu Y, Burykin A, Deem MW, Buchman TG. Predicting clinical physiology: a Markov chain model of heart rate recovery after spontaneous breathing trials in mechanically ventilated patients. J Crit Care 2009;24:347-61.

[60] Goldberger AL, Amaral LA, Hausdorff JM, Ivanov P, Peng CK, Stanley HE. Fractal dynamics in physiology: alterations with disease and aging. Proc Natl Acad Sci U S A 2002;99 Suppl 1:2466-72.

[61] Bien MY, Hseu SS, Yien HW, et al. Breathing pattern variability: a weaning predictor in postoperative patients recovering from systemic inflammatory response syndrome. Intensive Care Med 2004;30:241-7.

[62] Casaseca-de-la-Higuera P, Martin-Fernandez M, Alberola-Lopez C. Weaning from mechanical ventilation: a retrospective analysis leading to a multimodal perspective. IEEE Trans Biomed Eng 2006;53:1330-45.

[63] Ellenby MS, McNames J, Lai S, et al. Uncoupling and recoupling of autonomic regulation of the heart beat in pediatric septic shock. Shock 2001;16:274-7.

[64] Giraldo BF, Chaparro J, Ballesteros D, et al. Study of the respiratory pattern variability in patients during weaning trials. Conf Proc IEEE Eng Med Biol Soc 2004;6:3909-12.

[65] Godin PJ, Buchman TG. Uncoupling of biological oscillators: a complementary hypothesis concerning the pathogenesis of multiple organ dysfunction syndrome. Crit Care Med 1996;24:1107-16. 
[66] Van den Berghe GH. The neuroendocrine stress response and modern intensive care: the concept revisited. Burns 1999;25:7-16.

[67] Wysocki M, Cracco C, Teixeira A, et al. Reduced breathing variability as a predictor of unsuccessful patient separation from mechanical ventilation. Crit Care Med 2006;34:2076-83.

[68] Martich GD, Waldmann CS, Imhoff M. Clinical informatics in critical care. J Intensive Care Med 2004;19:154-63.

[69] Miller GA. The magical number seven plus or minus two: some limits on our capacity for processing information. Psychol Rev 1956;63:81-97.

[70] www.healthcare.philips.com/us_en/products/patient_monitoring/products/intellivue_patient_ monitors/index.wpd

[71] www.integra-ls.com/products/?product $=48$

[72] www.medcompare.com/details/15937/Camino-MPM-1-Multi-Parameter-Monitor.html

[73] www.somanetics.com/invos-system

[74] www.hemedex.com/Clinical Applications.htm

[75] www.cszmedical.com/products/hyper-hypthermia/Blanketrol3.htm 


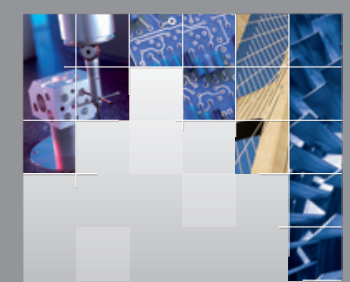

\section{Enfincering}
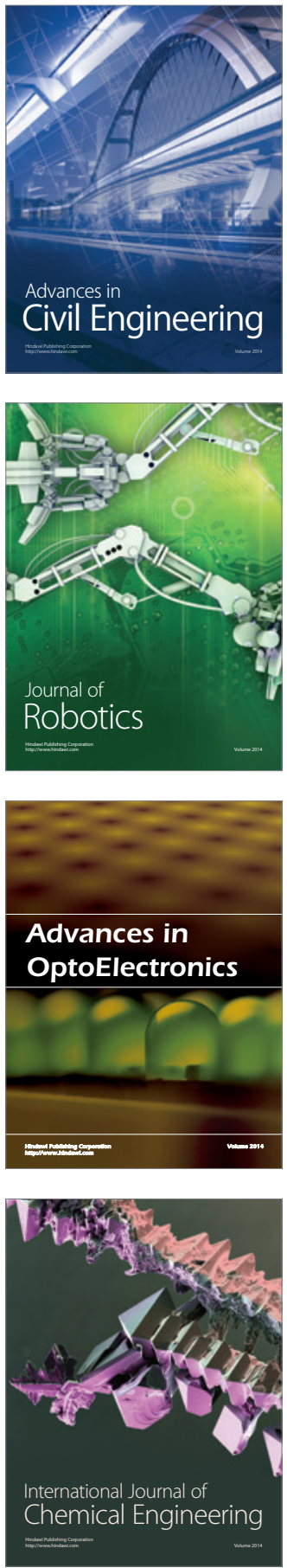

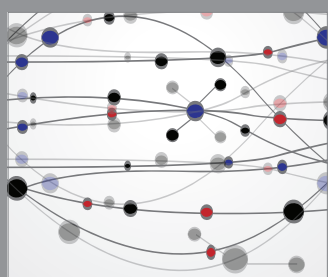

The Scientific World Journal

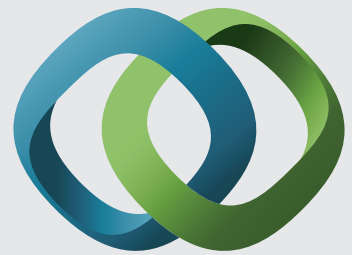

\section{Hindawi}

Submit your manuscripts at

http://www.hindawi.com
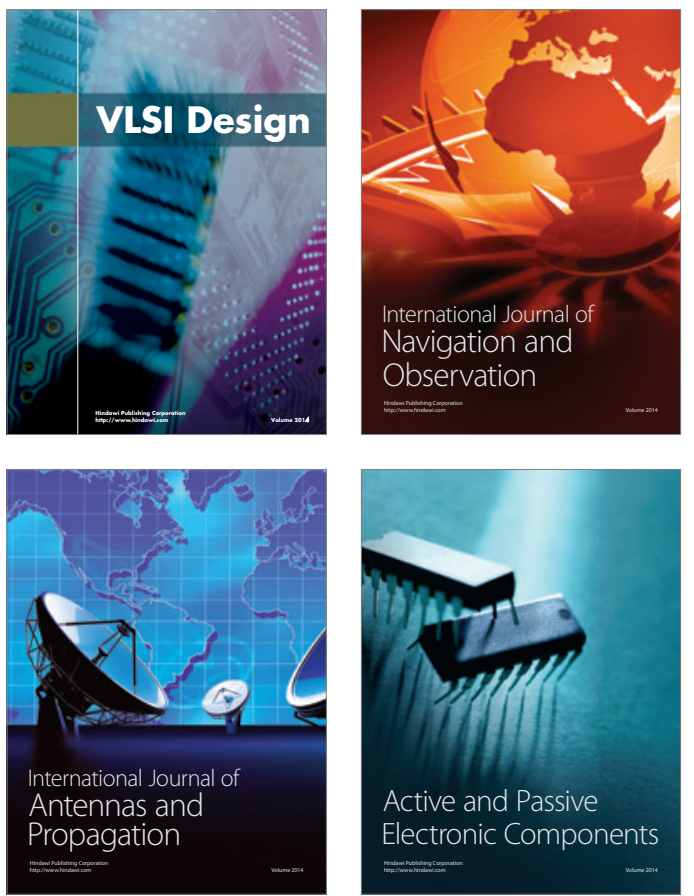
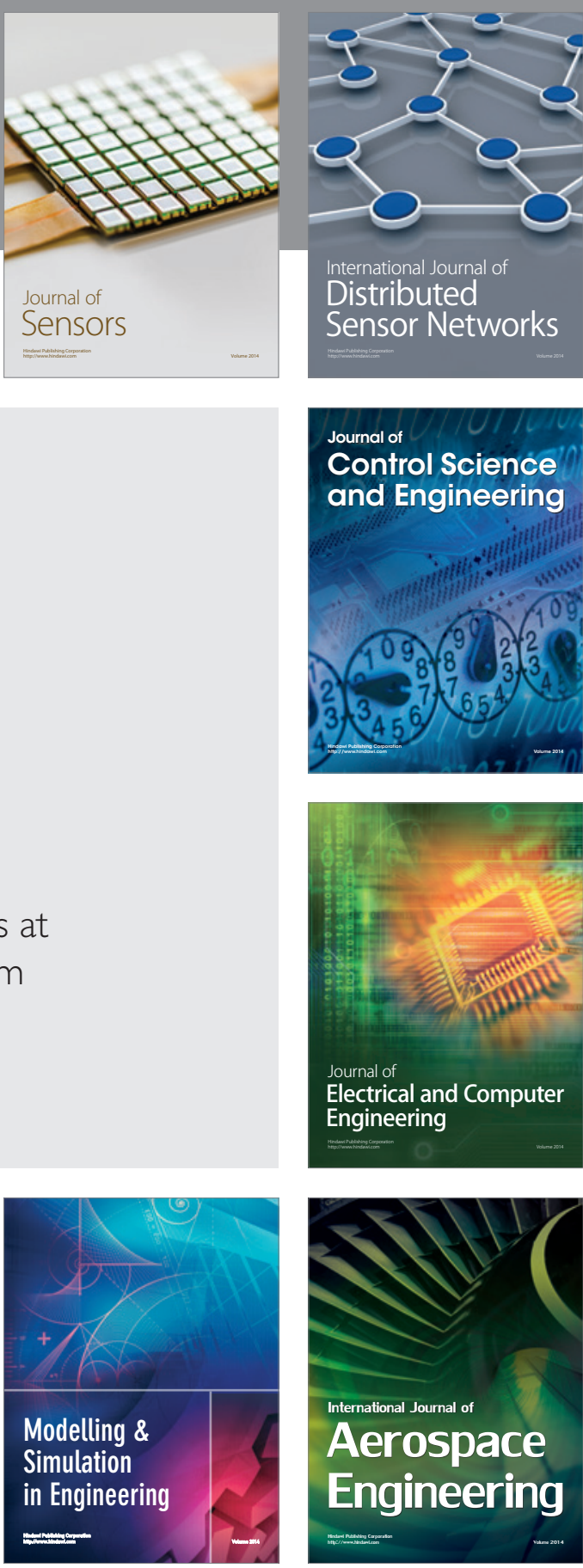

International Journal of

Distributed

Sensor Networks

Journal of

Control Science

and Engineering
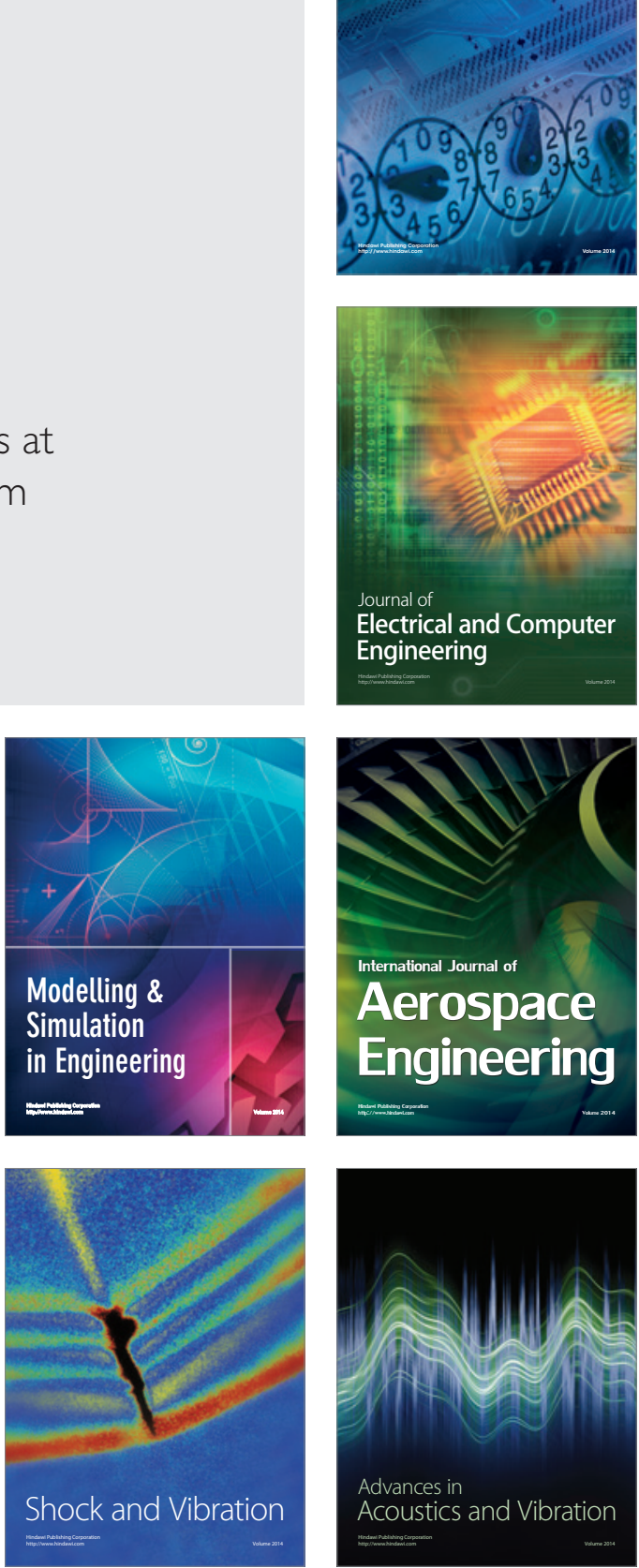